\title{
SERIES OF POSITIVE TERMS
}

\author{
G. BENNETT \\ Department of Mathematics, Indiana University, Bloomington, IN 47405, U.S.A. \\ E-mail: bennettg@indiana.edu
}

Let me begin with a couple of examples that may serve to explain my enthusiasm for series of positive terms.

EXAMPLE 1. I first became interested in this subject when I realized that an argument of Hardy could be adapted to give a new proof of Hilbert's Double Series Theorem.

In its final form Hilbert's result asserts that

$$
\sum_{j, k=1}^{\infty} \frac{x_{j} y_{k}}{j+k-1} \leq \frac{\pi}{\sin \left(\frac{\pi}{p}\right)}\left(\sum_{k=1}^{\infty} x_{k}^{p}\right)^{\frac{1}{p}}\left(\sum_{k=1}^{\infty} y_{k}^{p^{*}}\right)^{\frac{1}{p^{*}}}
$$

whenever $p>1$, and that the constant is best possible ([11], Theorem 323). An equivalent statement in more modern terms is that the Hilbert matrix is bounded on $\ell^{p}(p>1)$ with norm $\pi \operatorname{cosec}\left(\frac{\pi}{p}\right)$, i.e.,

$$
\|H\|_{p, p}=\frac{\pi}{\sin \left(\frac{\pi}{p}\right)} \quad(p>1),
$$

where $H$ denotes the Hilbert matrix whose entries are given by

$$
h_{j, k}=\frac{1}{j+k-1} \quad(j, k=1,2, \ldots) .
$$

Operator Theory, in Hilbert's time, was in a very rudimentary state, the best general result then available being

Frobenius's Theorem ([11], Theorem 275). A matrix, A, is bounded on $\ell^{p}(p>1)$ provided that

$$
R:=\sup _{j} \sum_{k=1}^{\infty}\left|a_{j, k}\right|<\infty
$$

2000 Mathematics Subject Classification: 26D15.

Key words and phrases: Hilbert's Inequality, Hardy's Inequality, Five Series Theorem, Heads/ Tails Option, Sneak-Out Principle.

The paper is in final form and no version of it will be published elsewhere. 
and

$$
C:=\sup _{k} \sum_{j=1}^{\infty}\left|a_{j, k}\right|<\infty
$$

Moreover, we then have

$$
\|A\|_{p, p} \leq C^{\frac{1}{p}} R^{\frac{1}{p^{*}}} .
$$

Hilbert's theorem caused quite a stir, because, aside from its great intrinsic beauty, it failed to fit in here. Frobenius's result is the first example of what we now call an interpolation theorem. Profound extensions were made by Riesz [21] and Thorin [22], but it wasn't until the appearance of Marcinkiewicz's "weak-type" result [16], that Hilbert's theorem finally succumbed to "proof by interpolation." Even then, the derivation was imperfect, because it failed to give the correct constant, $\pi \operatorname{cosec}\left(\frac{\pi}{p}\right)$.

In view of these remarks it comes as a great surprise to learn that

FrobeniUs's THEOREM $\Rightarrow$ HILBERT's.

Proof. We work with an auxiliary family of matrices, $H(\theta), 0<\theta<1$, whose entries are given by

$$
h_{j, k}(\theta)=\left(\begin{array}{c}
j+k-2 \\
j-1
\end{array}\right)(1-\theta)^{j-1} \theta^{k-1} \quad(j, k=1,2, \ldots) .
$$

It is plain that

$$
C(\theta)=\frac{1}{\theta} \quad \text { and } \quad R(\theta)=\frac{1}{1-\theta}
$$

so that, by Frobenius's theorem,

$$
\|H(\theta)\|_{p, p} \leq \theta^{-\frac{1}{p}}(1-\theta)^{-\frac{1}{p^{*}}} .
$$

On the other hand,

$$
h_{j, k}=\int_{0}^{1} h_{j, k}(\theta) d \theta
$$

so that

$$
\begin{aligned}
\|H\|_{p, p} & =\left\|\int_{0}^{1} H(\theta) d \theta\right\|_{p, p} \leq \int_{0}^{1}\|H(\theta)\|_{p, p} d \theta \\
& \leq \int_{0}^{1} \theta^{-\frac{1}{p}}(1-\theta)^{-\frac{1}{p^{*}}} d \theta=\Gamma\left(1-\frac{1}{p}\right) \Gamma\left(\frac{1}{p}\right) \\
& =\pi \operatorname{cosec}\left(\frac{\pi}{p}\right) .
\end{aligned}
$$

Hardy [10] gives a similar proof of his own inequality,

$$
\|C \mathbf{x}\|_{p, p} \leq p^{*}\|\mathbf{x}\|_{p} \quad(p>1),
$$

where $C$ is the Cesàro matrix with entries determined by

$$
c_{j, k}= \begin{cases}1 / j & \text { if } k \leq j \\ 0 & \text { if } k>j .\end{cases}
$$


The appropriate auxiliary matrices, however, were already in place (the familiar Euler matrices from classical Summability Theory) and their norms had previously been calculated by Bochner and Knopp (see [10], page 49). Hardy, moreover, chooses to work from scratch, in effect reproving Frobenius's theorem, and he thus misses the interpolatory nature of his result.

The basic idea - that of introducing an auxiliary family of matrices, then "averaging" against them - offers many interesting possibilities. It is used in Theorem 6 of [1], for example, to prove that

$$
\|H \mathbf{x}\|_{p} \leq \frac{\pi}{p^{*} \sin \left(\frac{\pi}{p}\right)}\|C \mathbf{x}\|_{p} \quad(p>1) .
$$

This shows that Hardy's inequality is better than Hilbert's.

EXAMPLE 2. My second example is an extrapolation result. It illustrates how, in proving certain $\ell^{p}$-inequalities, we may "forget about the $p . "$

The inequality in question is given by the following

TheOREM 1. If $p \geq 1$ and $\alpha p>1$, then

$$
\sum_{n=1}^{\infty}\left(\frac{1}{n^{\alpha}} \sum_{k=1}^{n}\left(k^{\alpha}-(k-1)^{\alpha}\right) x_{k}\right)^{p} \leq\left(\frac{\alpha p}{\alpha p-1}\right)^{p} \sum_{k=1}^{\infty} x_{k}^{p} .
$$

When $p=1,(13)$ reduces to

$$
\left(k^{\alpha}-(k-1)^{\alpha}\right) \sum_{n=k}^{\infty} \frac{1}{n^{\alpha}} \leq \frac{\alpha}{\alpha-1} \quad(\alpha>1),
$$

and our aim is to show how (13) follows from (14). (Inequality (14), of course, has got to be proved independently and this has been done by Cochran and Lee [7], Lemma 3.)

Proof of $(14) \Rightarrow$ (13). Hölder's inequality guarantees that

$$
\left(\sum_{k=1}^{n}\left(k^{\alpha}-(k-1)^{\alpha}\right) x_{k}\right)^{p} \leq\left(\sum_{k=1}^{n} k^{\alpha-\frac{1}{p}}-(k-1)^{\alpha-\frac{1}{p}}\right)^{p-1} \sum_{k=1}^{n} \frac{\left(k^{\alpha}-(k-1)^{\alpha}\right)^{p} x_{k}^{p}}{\left(k^{\alpha-\frac{1}{p}}-(k-1)^{\alpha-\frac{1}{p}}\right)^{p-1}} .
$$

The first series on the right telescopes, and we deduce that

$$
\begin{aligned}
\sum_{n=1}^{\infty}\left(\frac{1}{n^{\alpha}} \sum_{k=1}^{n}\left(k^{\alpha}-(k-1)^{\alpha}\right) x_{k}\right)^{p} & \leq \sum_{n=1}^{\infty} \frac{1}{n^{\alpha+\frac{1}{p^{*}}}} \sum_{k=1}^{n} \frac{\left(k^{\alpha}-(k-1)^{\alpha}\right)^{p} x_{k}^{p}}{\left(k^{\alpha-\frac{1}{p}}-(k-1)^{\alpha-\frac{1}{p}}\right)^{p-1}} \\
& =\sum_{k=1}^{\infty} x_{k}^{p} \frac{\left(k^{\alpha}-(k-1)^{\alpha}\right)^{p}}{\left(k^{\alpha-\frac{1}{p}}-(k-1)^{\alpha-\frac{1}{p}}\right)^{p-1}} \sum_{n=k}^{\infty} \frac{1}{n^{\alpha+\frac{1}{p^{*}}}} \\
& \leq\left(\frac{\alpha p}{\alpha p-1}\right)^{p} \sum_{k=1}^{\infty} x_{k}^{p} .
\end{aligned}
$$

The last step needs to be explained. It demands that

$$
\sum_{n=k}^{\infty} \frac{1}{n^{\alpha+\frac{1}{p^{*}}}} \leq\left(\frac{\alpha p}{\alpha p-1}\right)^{p} \frac{\left(k^{\alpha-\frac{1}{p}}-(k-1)^{\alpha-\frac{1}{p}}\right)^{p-1}}{\left(k^{\alpha}-(k-1)^{\alpha}\right)^{p}},
$$

and we attempt to justify this by invoking inequality (14) with $\alpha$ replaced by $\alpha+\frac{1}{p^{*}}$ : 


$$
\sum_{n=k}^{\infty} \frac{1}{n^{\alpha+\frac{1}{p^{*}}}} \leq \frac{\alpha+\frac{1}{p^{*}}}{\alpha-\frac{1}{p}} \frac{1}{k^{\alpha+\frac{1}{p^{*}}}-(k-1)^{\alpha+\frac{1}{p^{*}}}} .
$$

All is well if the right-hand side of $\left(14^{\prime}\right)$ is smaller than the right-hand side of (15). This amounts to checking that a rather intimidating estimate,

$$
\left(\frac{k^{\alpha}-(k-1)^{\alpha}}{\alpha}\right)^{p} \leq\left(\frac{k^{\alpha-\frac{1}{p}}-(k-1)^{\alpha-\frac{1}{p}}}{\alpha-\frac{1}{p}}\right)^{p-1} \frac{k^{\alpha+\frac{1}{p^{*}}}-(k-1)^{\alpha+\frac{1}{p^{*}}}}{\alpha+\frac{1}{p^{*}}}
$$

is valid whenever $p>1, \alpha p>1$, and $k=1,2, \ldots$.

But (16) may be rephrased as

$$
\left(\int_{k-1}^{k} \theta^{\alpha-1} d \theta\right)^{p} \leq\left(\int_{k-1}^{k} \theta^{\alpha-\frac{1}{p}-1} d \theta\right)^{p-1} \int_{k-1}^{k} \theta^{\alpha+\frac{1}{p^{*}}-1} d \theta
$$

and, since

$$
\alpha-1=\frac{1}{p^{*}}\left(\alpha-\frac{1}{p}-1\right)+\frac{1}{p}\left(\alpha+\frac{1}{p^{*}}-1\right),
$$

our good friend, Hölder, saves the day.

Theorem 1 contains Hardy's inequality as a special case $(\alpha=1)$ and our argument may be viewed as offering a new approach to his result. It also solves a problem raised in [3], where inequality (13) first appeared, but with the added restriction: $\alpha \geq 1$. The extension given here, to all $\alpha>1 / p$, allows us to deduce the following version of Carleman's inequality. This answers a question of Cochran and Lee (see [3], page 40).

Corollary 1. If $\alpha>0$, then

$$
\sum_{n=1}^{\infty}\left(\prod_{k=1}^{n} x_{k}^{\left(k^{\alpha}-(k-1)^{\alpha}\right)}\right)^{\frac{1}{n^{\alpha}}} \leq e^{\frac{1}{\alpha}} \sum_{k=1}^{\infty} x_{k} .
$$

Proof. Replace $x_{k}$ in (13) by $x_{k}^{1 / p}$ and make $p \rightarrow \infty$.

This elegant result which reduces to Carleman's inequality ([11], Theorem 334) when $\alpha=1$, was given by Love in his plenary address at the Hardy, Littlewood, Polya fest, [15].

There are no obvious connections between the work of Professor Orlicz and series of positive terms. A path can be woven, however, from one of his earliest papers, and it goes something like this:

$$
\underset{\text { Littlewood }}{\text { Orlicz }} \longrightarrow \text { Grothendieck } \longrightarrow \begin{gathered}
\text { Lindenstrauss } \\
\text { Pełczyński }
\end{gathered} \longrightarrow \text { Maurey. }
$$

Orlicz was the first to study unconditionally convergent series in Banach spaces. His earliest, and perhaps most famous paper [18], dealt with abstract spaces, but we take as our starting point his work on the $L^{p}$-spaces, [19] and [20]. Littlewood proved a similar result a few years earlier, but the emphasis of his paper, [14], is quite different. In any event, their work lay dormant for many years until it was picked up again by Grothendieck in his seminal report on tensor products [9]. The theorems of Orlicz and Littlewood actually provided the first examples in Grothendieck's theory of "absolutely summing operators." 
Grothendieck's work also lay dormant for some time, and it wasn't until the appearance of the highly influential paper of Lindenstrauss and Pełczyński [13] that his results became widely known. It can fairly be said that the appearance of [13] signaled the birth of modern Banach space theory. I was in graduate school at the time yet I remember still the great excitement that it caused. Absolutely summing operators seemed to be cropping up all over the place, along with their attendant "factorization theory."

It was the remarkable thesis of Maurey [17], however, that showed how factorization theory could be applied in an elementary setting. His ideas were used in [2] to solve the $\ell^{p} \rightarrow \ell^{q}$ mapping problem for matrices of the type

$$
a_{j, k}= \begin{cases}a_{j} b_{k} & \text { if } k \leq j \\ 0 & \text { if } k>j\end{cases}
$$

A simple factorization problem turned out to be of central importance: when can a sequence $\mathrm{x}$ be factorized,

$$
\mathbf{x}=\mathbf{y} \cdot \mathbf{z} \quad(\text { coordinatewise product })
$$

in such a way that

$$
\mathbf{y} \in \ell^{p}
$$

and

$$
\sum_{k=1}^{n} z_{k}^{q} \leq \sum_{k=1}^{n} a_{k} \quad(n=1,2, \ldots) ?
$$

( $p, q$ and a are here supposed fixed.)

This problem is studied extensively in [3], wherein the classical inequalities of Hilbert, Hardy et al. are replaced by identities in such a way that nothing is lost. Several of the problems left open in [3] were solved by Grosse-Erdmann in his encyclopaedic paper [8]. (Any serious student of Series of Positive Terms must consult his work!)

Grosse-Erdmann visited Bloomington for the academic year, 2000-2001, and the remainder of my talk is part of the joint work we did then. In particular, I wish to advertise three new techniques for handling series of positive terms that have emerged from our investigations.

Our main result ([5], Theorem 2) gives a complete characterization of the "weights", $\mathbf{a}$ and $\mathbf{b}$, for which the inequality

$$
\left(\sum_{n=1}^{\infty} a_{n}\left(\sum_{k=1}^{n} x_{k}\right)^{q}\right)^{\frac{1}{q}} \leq C\left(\sum_{k=1}^{\infty} b_{k} x_{k}^{p}\right)^{\frac{1}{p}}
$$

holds whenever $\mathbf{x}$ is a decreasing sequence (of positive terms). Here $p$ and $q$ are fixed parameters, with $0<p, q<\infty$, and the constant $C$ is independent of $\mathbf{x}$.

The theorem has interesting applications to Lorentz spaces, [6], but, unfortunately, it is too complicated to be described in detail here. There is, however, an analogous inequality, much simpler than (24),

$$
\left(\sum_{n=1}^{\infty} a_{n} x_{n}^{q}\right)^{\frac{1}{q}} \leq C\left(\sum_{n=1}^{\infty} b_{n} x_{n}^{p}\right)^{\frac{1}{p}}
$$


that can be discussed instead. $((25)$ actually arises in an essential way in our analysis of (24).)

We find ([4], Theorem 3), in case $p \leq q$, that (25) holds (for all decreasing sequences $\mathrm{x})$ precisely when

$$
\left(\sum_{k=1}^{n} a_{k}\right)^{\frac{1}{q}} \leq C\left(\sum_{k=1}^{n} b_{k}\right)^{\frac{1}{p}} \quad(n=1,2, \ldots) .
$$

(This provides another example of an extrapolation result, wherein we now "ignore the x.")

The complementary case ([4], Theorem 4) is much more interesting. We find, when $p>q$, that (25) holds if and only if a admits a factorization:

$$
a_{n}=c_{n} d_{n} \quad(n=1,2, \ldots)
$$

with

$$
\sum_{n=1}^{\infty} c_{n}^{\frac{p}{p-q}} \leq C^{\frac{p q}{p-q}}
$$

and

$$
\sum_{k=1}^{n} d_{n}^{\frac{p}{q}} \leq \sum_{k=1}^{n} b_{k} \quad(n=1,2, \ldots) .
$$

The factorization, (27)-(29), it will be noticed, is the same as that which arose in the analysis of the classical inequalities, namely, (21)-(23). Based upon our respective experiences with [8] and [3], both Grosse-Erdmann and I were able to come up promptly with several alternatives to inequality (25). The plot thickened, so to speak, when we compared our solutions ... and found that none of them matched!

After much deliberation we were forced to concede that neither of us had erred, and that the following remarkable equivalence had to be valid.

The Five Series Theorem ([4], Theorem 1). If $p>0$ and $\mathbf{a}$ and $\mathbf{b}$ are sequences of positive terms with

$$
A_{n}=a_{1}+\cdots+a_{n} \text { and } B_{n}=b_{1}+\cdots+b_{n},
$$

then the following conditions are equivalent.

(i) $\quad \sum_{n=1}^{\infty} a_{n}\left(\sum_{k=n}^{\infty} \frac{a_{k}}{B_{k}}\right)^{p}<\infty$

$$
\sum_{n=1}^{\infty} b_{n}\left(\sum_{k=n}^{\infty} \frac{a_{k}}{B_{k}}\right)^{p+1}<\infty
$$

(iii) $\quad \sum_{n=1}^{\infty} a_{n} \sup _{k \geq n}\left(\frac{A_{k}}{B_{k}}\right)^{p}<\infty$

$$
\sum_{n=1}^{\infty} b_{n} \sup _{k \geq n}\left(\frac{A_{k}}{B_{k}}\right)^{p+1}<\infty
$$

(v) $\quad \sum_{n=1}^{\infty} a_{n}\left(\frac{A_{n}}{B_{n}}\right)^{p}<\infty$.

The theorem has a couple of striking features. First, of course, it is a result of great generality, yet one cannot help but be offended by the appalling lack of symmetry engendered by the omission of the "obvious" sixth condition 


$$
\sum_{n=1}^{\infty} b_{n}\left(\frac{A_{n}}{B_{n}}\right)^{p+1}<\infty .
$$

It turns out that (vi) is weaker than (i)-(v), and that the converse implication fails, even when an additional hypothesis is applied:

$$
\left.\begin{array}{l}
\sum_{n=1}^{\infty} b_{n}\left(\frac{A_{n}}{B_{n}}\right)^{p+1}<\infty \\
\sup _{n} \frac{A_{n}^{p+1}}{B_{n}^{p}}<\infty
\end{array}\right\} \nRightarrow \sum_{n=1}^{\infty} a_{n}\left(\frac{A_{n}}{B_{n}}\right)^{p}<\infty .
$$

This failure is very surprising because the analogous implication for integrals does hold ([4], Proposition 1).

We have been unable to restore symmetry to the Five Series Theorem, the rather unwieldy hypothesis,

$$
\sum_{n=1}^{\infty} b_{n+1} \frac{B_{n}}{B_{n+1}}\left(\frac{A_{n}}{B_{n}}\right)^{p+1}<\infty,
$$

coming closest to achieving our goal. Condition ( $\left.\mathrm{vi}^{\prime}\right)$, too, is weaker than those of the Five Series Theorem, but, unlike (vi), it turns out to be equivalent in case $B_{n} \rightarrow \infty$. The appearance of $\left(\mathrm{vi}^{\prime}\right)$ in our analysis of inequality (24) is unavoidable and it produces many technical difficulties.

Another technique that has emerged from our work in [4] is a sort of duality principle for series of positive terms. It provides a very precise connection between

$$
\text { the rate of growth of the partial sums (Heads) of one series }
$$

and

$$
\text { the rate of decay of the remainder terms (Tails) of another. }
$$

The two series, of course, have got to be related, as specified below, but the relationship can be quite lax, there being considerable flexibility in the selection of various parameters $(\alpha, \beta, \mathbf{a}$ and $\mathbf{b})$. The theorem, in effect, offers us a choice: should we worry about controlling (32), or should we attempt to estimate (33) instead? We call this the

Heads/Tails Option ([4], Theorem 13). If $\alpha>0$ and $\beta<0$, then

$$
\sup _{n} B_{n}^{\alpha} \sum_{k=n}^{\infty} \frac{a_{k}}{B_{k}^{\alpha}}<\infty
$$

if and only if

$$
\sup _{n} B_{n}^{\beta} \sum_{k=1}^{n} \frac{a_{k}}{B_{k}^{\beta}}<\infty .
$$

Moreover, we have

$$
S_{\alpha} \leq \frac{\alpha-\beta}{\alpha} S_{\beta}
$$


and

$$
S_{\beta} \leq \frac{\beta-\alpha}{\beta} S_{\alpha}
$$

where $S_{\alpha}, S_{\beta}$ denote the respective suprema in (34) and (35). The constants in (36) and (37) are best possible.

A surprising consequence of the Heads/Tails Option is that (34) is independent of $\alpha$. In fact, it can be shown that

$$
S_{\alpha^{\prime}} \leq S_{\alpha} \leq \frac{\alpha^{\prime}}{\alpha} S_{\alpha^{\prime}}
$$

whenever $\alpha^{\prime}>\alpha>0$ and, similarly, that

$$
S_{\beta^{\prime}} \leq S_{\beta} \leq \frac{\beta^{\prime}}{\beta} S_{\beta^{\prime}}
$$

whenever $\beta^{\prime}>\beta>0$. The constants in (38) and (39) are best possible.

If we set $\beta=-1$ and $a_{k}=b_{k} / B_{k}$, we see that (35) is automatically satisfied, with $S_{\beta}=1$. It then follows, from $(35) \Rightarrow(34)$, that

$$
\sum_{k=n}^{\infty} \frac{b_{k}}{B_{k}^{a+1}} \leq \frac{\alpha+1}{\alpha} \frac{1}{B_{n}^{\alpha}} \quad(\alpha>0)
$$

whenever $\mathbf{b}$ is an arbitrary sequence of positive terms. This lends precision to an old assertion of Dini ([12], page 299):

$$
\sum_{k=1}^{\infty} b_{k}=\infty \Rightarrow \sum_{k=1}^{\infty} \frac{b_{k}}{B_{k}^{\alpha+1}}<\infty \quad(\alpha>0) .
$$

Inequality (40) may be proved directly, via the Integral Test, and it has been re-discovered by many authors. That the constant $\frac{\alpha+1}{\alpha}$ is best possible, however, (a fact suggested by the Heads/Tails Option) may well be a new observation.

Our third technique is again concerned with equivalence - this time of the two series

$$
\sum_{n=1}^{\infty} a_{n}\left(\sum_{k=n}^{\infty} A_{k}^{\alpha} x_{k}\right)^{p} \approx \sum_{n=1}^{\infty} a_{n} A_{n}^{\alpha p}\left(\sum_{k=n}^{\infty} x_{k}\right)^{p} .
$$

In other words, we want to determine precisely when it is possible to "sneak" the $A_{k}^{\alpha}$ outside the inner sum. We assume that $p>0$ and that $\mathbf{a}$ and $\mathbf{x}$ are sequences of positive terms. It is plain, then, that

$$
\sum_{n=1}^{\infty} a_{n} A_{n}^{\alpha p}\left(\sum_{k=n}^{\infty} x_{k}\right)^{p} \leq \sum_{n=1}^{\infty} a_{n}\left(\sum_{k=n}^{\infty} A_{k}^{\alpha} x_{k}\right)^{p}
$$

when $\alpha \geq 0$, and that the inequality reverses direction when $\alpha \leq 0$.

The trivial estimate (43) was used several times in [5], but only ever as a last resort. Our goal in [5], after all, was to find necessary and sufficient conditions (for inequality (24) to hold), and we had to be economical at every stage. It was striking how often (43) gave the correct result, and this is what led us to suspect that a converse inequality might 
be valid:

$$
\sum_{n=1}^{\infty} a_{n}\left(\sum_{k=n}^{\infty} A_{k}^{\alpha} x_{k}\right)^{p} \leq K(\alpha, p) \sum_{n=1}^{\infty} a_{n} A_{n}^{\alpha p}\left(\sum_{k=n}^{\infty} x_{k}\right)^{p}
$$

(with reversal when $\alpha \leq 0$ ). Here $\alpha$ and $p$ are fixed, as is the constant $K(\alpha, p)$, and the estimate is to hold for all positive sequences $\mathbf{a}$ and $\mathbf{x}$.

It turns out that (44) is valid (with reversal when $\alpha \leq 0$ ) if and only if $\alpha>-1 / p$. We call this the Sneak-Out Principle.

The equivalence of the two series (42) may seem like no big deal, but the fact that it always holds (when $\alpha p>-1$ ) allows us to "sneak-out" or "sneak-in" whenever we please. What originally seemed to be very daring steps, taken in some of the more technical arguments of [5], have now become matters of routine. This, alas, will probably be lost on the naive reader of [5], because the Sneak-Out Principle is often applied there in its trivial direction, (43).

The three techniques described here are very simple, to be sure, but a great deal of thought went into their formulation. Some evidence for their validity was provided by our work in [5] and [6], where they are applied repeatedly, but always in disguise. It was, in fact, only after we had scored several successes, by what seemed like ad hoc methods, that we recognized the possibilities involved.

There are intimate connections between all three of our techniques. The best value of the constant in (44), for example, when $0<p \leq 1$ and $\alpha \geq 0$, is known to be ([4], Theorem 6)

$$
K(\alpha, p)=\alpha p+1
$$

Replacing $\alpha$ by $\alpha / p$ and $x_{k}$ by $x_{k}^{1 / p}$ in (44), then making $p \rightarrow 0$, gives

$$
\sum_{n=1}^{\infty} a_{n} \sup _{k \geq n}\left(A_{k}^{\alpha} x_{k}\right) \leq(\alpha+1) \sum_{n=1}^{\infty} a_{n} A_{n}^{\alpha} \sup _{k \geq n} x_{k} .
$$

This reduces to what is perhaps the most striking of the implications of the Five Series Theorem, (v) $\Rightarrow$ (iii), when $x_{k}=B_{k}^{-\alpha}$.

In the final analysis, however, it is in the simplicity of our techniques that their power really lies, for, unencumbered by any hypotheses, they may be applied with abandon.

\section{References}

[1] G. Bennett, Lower bounds for matrices, Lin. Algebra Appl. 82 (1986), 81-98.

[2] G. Bennett, Some elementary inequalities III, Quart. J. Math. Oxford Ser. (2) 42 (1991), 149-174.

[3] G. Bennett, Factorizing the classical inequalities, Mem. Amer. Math. Soc. 120 (1996), no. 576.

[4] G. Bennett and K.-G. Grosse-Erdmann, On series of positive terms, Houston J. Math., to appear.

[5] G. Bennett and K.-G. Grosse-Erdmann, Weighted Hardy inequalities for decreasing sequences and functions, preprint. 
[6] G. Bennett and K.-G. Grosse-Erdmann, Some remarks on Lorentz spaces, preprint.

[7] J. A. Cochran and C. S. Lee, Inequalities related to Hardy's and Heinig's, Math. Proc. Camb. Phil. Soc. 96 (1) (1984), 1-7.

[8] K.-G. Grosse-Erdmann, The blocking technique, weighted mean operators and Hardy's inequality, Lecture Notes in Math. 1679, Springer, Berlin, 1998.

[9] A. Grothendieck, Résumé de la théorie métrique des produits tensoriels topologiques, Bol. Soc. Mat. São Paulo 8 (1953/6), 1-79.

[10] G. H. Hardy, An inequality for Hausdorff means, J. London Math. Soc. 18 (1943), 46-50.

[11] G. H. Hardy, J. E. Littlewood and G. Pólya, Inequalities, Cambridge University Press, Cambridge, 1967.

[12] K. Knopp, Theorie und Anwendungen der unendlichen Reihen, Springer, Berlin, 1964.

[13] J. Lindenstrauss and A. Pełczyński, Absolutely summing operators in $\mathcal{L}^{p}$-spaces and their applications, Studia Math. 29 (1968), 275-326.

[14] J. E. Littlewood, On bounded bilinear forms in an infinite number of variables, Quart. J. Math. Oxford 1 (1930), 164-174.

[15] E. R. Love, Inequalities related to Carleman's inequality, in: Inequalities (Birmingham, 1987), Lecture Notes in Pure and Appl. Math. 129, Dekker, New York, 1991, 135-141.

[16] J. Marcinkiewicz, Sur l'interpolation d'opérations, C. R. Acad. Sci. Paris 208 (1939), 12721273.

[17] B. Maurey, Théorèmes de factorisation pour les opérateurs linéaires à valeurs dans les espaces $L^{p}$, Astérisque 11 (1974).

[18] W. Orlicz, Beiträge zur Theorie der Orthogonalentwicklungen II, Studia Math. 1 (1929), 241-255.

[19] W. Orlicz, Über unbedingte Konvergenz in Funktionenräumen I, Studia Math. 4 (1933), 33-37.

[20] W. Orlicz, Über unbedingte Konvergenz in Funktionenräumen II, Studia Math. 4 (1933), 41-47.

[21] M. Riesz, Sur les maxima des formes bilinéaires et sur les fonctionnelles linéaires, Acta Math. 49 (1926), 465-497.

[22] G. O. Thorin, An extension of a convexity theorem due to M. Riesz, Kungl. Fys. Saell. i Lund For. 8 (1939), \#14. 